\title{
BROWNIAN MOTION IN A CLOSED CONVEX POLYGON WITH NORMAL REFLECTION
}

\author{
L.L. Helms \\ University of Illinois at Urbana-Champaign, Department of Mathematics \\ 273 Altgeld Hall, 1409 West Green Street, Urbana, IL 61801, U.S.A.
}

\begin{abstract}
Brownian motion on a closed convex polygon in the plane with normal reflection at boundary points, with the exception of the vertices, is constructed. This is done by constructing a Feller semigroup whose generator is a closed extension of the Laplacian with domain consisting of functions for which the normal derivative vanishes at the non-vertex boundary points.
\end{abstract}

\section{Introduction}

Recent papers have dealt with the construction of Brownian motion on a convex subset of $\mathbf{R}^{n}$ which undergoes normal reflection upon reaching the boundary or, more generally, undergoes oblique reflection upon reaching the boundary. Tanaka [7] has constructed a pathwise solution of a stochastic differential equation with normal reflection at the boundary by modifying the paths of $n$-dimensional Brownian motion on $\mathbf{R}^{n}$. Varadhan and Williams [9] have investigated Brownian motion on a wedge in $\mathbf{R}^{2}$ with oblique reflection at the boundary. Williams [10] has also constructed a Brownian motion on a convex polyhedron in $\mathbf{R}^{n}$ from which the vertices, among other boundary points, have been removed with oblique reflection at the remaining boundary points. This result precludes starting the Brownian motion at one of the vertices. In this paper we will construct a Brownian motion on a compact convex polygon in $\mathbf{R}^{2}$ with normal reflection at the non-vertex boundary points. The results of this paper are not immediately applicable to a three-dimensional polyhedron due to some unresolved problems associated with a smoothing operation on the boundary of the polyhedron and a description of the resulting boundary using a local coordinate system.

If $S$ is any open subset of $\mathbf{R}^{2}$ and $k$ is a nonnegative integer or $\infty, C^{k}(S)$ will denote the set of functions (= real-valued functions) on $S$ all of whose derivatives of order less than or equal to $k$ are continuous on $S$ and $C^{k}(\bar{S})$ will denote the set of functions in $C^{k}(S)$ all of whose derivatives of order less than or equal to $k$ have continuous extensions to $\bar{S} . C_{0}^{k}(S)$ and $C_{0}^{k}(\bar{S})$ will denote subspaces of $C^{k}(S)$ and $C^{k}(\bar{S})$, respectively, consisting of functions with compact support in $S$. As usual, $C(\bar{S})$ will denote the set of bounded continuous functions on $\bar{S}$ with the supremum norm $|\cdot|$.

1991 Mathematics Subject Classification: Primary 60J25, 60J35, 60J65. 
Consider a bounded open convex subset $\Omega$ of $\mathbf{R}^{2}$ whose boundary is a polygon. Let $E$ denote the set of vertices of the polygon. Consider a fixed $\mu \in(0,1)$ and the Laplacian operator $\Delta$ acting on $u \in C^{2}(\bar{\Omega})$ with domain

$$
\mathscr{D}(\Delta) \subset\left\{u \in C^{0, \mu}(\bar{\Omega}) \cap C^{2}(\Omega): \Delta u \in C^{0, \mu}(\bar{\Omega}) \text { and } \frac{\partial u}{\partial \nu}=0 \text { on } \partial \Omega \backslash E\right\}
$$

where $\partial u / \partial \nu, \nu$ the outer unit normal, denotes the normal derivative of $u$ at points of $\partial \Omega \backslash E$ and $C^{0, \mu}(\bar{\Omega})$ is the Banach space of Hölder continuous functions of exponent $\mu$. The precise definition of $\mathscr{D}(\Delta)$ is given in Section 3. We will show that there is a Feller semigroup $\left\{T_{t}: t \geq 0\right\}$ on $C(\bar{\Omega})$ whose infinitesimal generator is an extension of the operator $\Delta$. If we can show that

(i) $\mathscr{D}(\Delta)$ is dense in $C(\bar{\Omega})$

(ii) $\Delta$ is dissipative

(iii) the range of $\Delta-\lambda$ is dense in $C(\bar{\Omega})$ for some $\lambda>0$,

then it follows that $\Delta$ is closable and its minimal closed extension $A$ generates a Feller semigroup $\left\{T_{t}: t \geq 0\right\}$ on $C(\bar{\Omega})$ (c.f. [1], p. 16). Condition (ii) means that $|\lambda u-\Delta u| \geq \lambda|u|$ for all $\lambda>0$ and $u \in \mathscr{D}(\Delta)$.

\section{Preliminaries}

The space $C^{k}(\bar{S})$ introduced above is a Banach space with norm $|\cdot|_{k, S}$ (see [2], p. 52 for definition of this norm). We will also need the spaces $C^{k, \alpha}(S), 0<\alpha<1$, $k \geq 0$, consisting of functions on $S$ all of whose derivatives of order less than or equal to $k$ are Hölder continuous on $S$ with exponent $\alpha$. The Hölder spaces $C^{k, \alpha}(\bar{S}), 0<\alpha<1, k \geq 0$, consist of functions on $S$ all of whose derivatives of order less than or equal to $k$ are Hölder continuous on $S$ with exponent $\alpha$ and have continuous extensions to $\bar{S}$. The $C^{k, \alpha}(\bar{S})$ are Banach spaces with norms $|\cdot|_{k, \alpha, S}$ as defined in [2]. We can allow $\alpha=0$ if we put $C^{k, 0}(S)=C^{k}(S)$ and $C^{k, \alpha}(\bar{S})=C^{k}(\bar{S})$. For $p \geq 1$ and $k$ a nonnegative integer, $W^{k, p}(S)$ will denote the Sobolev space of functions whose weak derivatives of order less than or equal to $k$ are $p$-integrable with norm $\|\cdot\|_{k, p, S}$ as defined in [2]. For functions $u$ on $S$ we will let $D_{i} u=\partial u / \partial x_{i}, D_{i j} u=\partial^{2} u / \partial x_{i} \partial x_{j}$, etc. If $\beta=\left(\beta_{1}, \beta_{2}\right)$ where each $\beta_{i}$ is a nonnegative integer and $|\beta|=\beta_{1}+\beta_{2}, D^{\beta} u=\partial^{|\beta|} u / \partial x_{1}^{\beta_{1}} \partial x_{2}^{\beta_{2}}$.

In order to approximate the region $\Omega$ by a region with a smooth boundary, we will employ a mollifier $\varrho: \mathbf{R} \rightarrow \mathbf{R}$ defined by

$$
\varrho(x)= \begin{cases}c \exp \left(\frac{1}{x^{2}-1}\right) & \text { for }|x|<1 \\ 0 & \text { for }|x| \geq 1\end{cases}
$$


where $c$ is a constant chosen so that $\varrho$ is a probability density. For $\varepsilon>0$ and a locally integrable function $f: \mathbf{R} \rightarrow \mathbf{R}$ let

$$
J_{\varepsilon} f(x)=\frac{1}{\varepsilon} \int_{|x-y| \leq \varepsilon} \varrho\left(\frac{x-y}{\varepsilon}\right) f(y) d y=\int_{|y| \leq 1} \varrho(y) f(x-\varepsilon y) d y .
$$

Then (i) if $f \geq 0, J_{\varepsilon} f \geq 0$, (ii) $J_{\varepsilon} f \in C^{\infty}(\mathbf{R})$, (iii) if $f$ is linear on $(x-\varepsilon, x+\varepsilon)$, then $J_{\varepsilon} f(x)=f(x)$, and (iv) if $f$ is convex on $\mathbf{R}$, then $J_{\varepsilon} f$ is convex on $\mathbf{R}$.

We will illustrate how the $J_{\varepsilon}$ operator can be used to smoothen the boundary of $\Omega$ in such a way that most of the smooth part is left intact by smoothing the boundary of the region $\Omega^{\prime}=\{(x, y): y>|x|\} \subset \mathbf{R}^{2}$. Let $f(x)=|x|, x \in \mathbf{R}$. Note that $J_{\varepsilon / \sqrt{2}} f$ is convex and a $C^{\infty}(\mathbf{R})$ function. Letting $\Omega_{\varepsilon}^{\prime}=\{(x, y): y>$ $\left.J_{\varepsilon / \sqrt{2}} f(x)\right\} \subset \mathbf{R}^{2}$, it is easily seen that $\Omega_{\varepsilon}^{\prime} \subset \Omega^{\prime}, \Omega^{\prime} \backslash \Omega_{\varepsilon}^{\prime} \subset N((0,0), \varepsilon)$, and that the symmetric difference $\partial \Omega^{\prime} \Delta \partial \Omega_{\varepsilon}^{\prime} \subset N((0,0), \varepsilon)$, the latter meaning that the $J_{\varepsilon / \sqrt{2}}$ smoothing operation does not affect the boundary of $\Omega^{\prime}$ outside an $\varepsilon$-neighborhood of the vertex $(0,0)$ of $\Omega^{\prime}$.

Returning to the region $\Omega$ with polygonal boundary, a covering of $\bar{\Omega}$ by a collection of open sets $\left\{W_{r}: 1 \leq r \leq M\right\}$ can be constructed with the following properties: (i) There is a subcollection $\left\{W_{r}: 1 \leq r \leq M_{0}\right\}$ which covers $\partial \Omega$ such that for each $r$ there are numbers $\alpha_{r}>0, \beta_{r}>0$, and a map $\left(x_{r 1}, x_{r 2}\right): \Delta_{r} \times \mathbf{R} \rightarrow$ $\mathbf{R}^{2}$, where $\Delta_{r}=\left(-\alpha_{r}, \alpha_{r}\right)$, and continuous piecewise linear functions $a_{r}: \Delta_{r} \rightarrow \mathbf{R}$ such that each point $x=\left(x_{1}, x_{2}\right) \in \partial \Omega$ can be represented by $x=\left(x_{1}, a_{r}\left(x_{1}\right)\right)$, $x_{1} \in \Delta_{r}$, for some $r=1, \ldots, M_{0}$, and in such a way that

$$
\begin{gathered}
\left\{\left(x_{r 1}, x_{r 2}\right) \in \mathbf{R}^{2}: x_{r 1} \in \Delta_{r}, a_{r}\left(x_{r 1}\right)<x_{r 2}<a_{r}\left(x_{r 1}\right)+\beta_{r}\right\} \subset \Omega \\
\left\{\left(x_{r 1}, x_{r 2}\right) \in \mathbf{R}^{2}: x_{r 1} \in \Delta_{r}, a_{r}\left(x_{r 1}\right)-\beta_{r}<x_{r 2}<a_{r}\left(x_{r 1}\right)\right\} \subset \bar{\Omega}^{c}
\end{gathered}
$$

and

$$
W_{r}=\left\{\left(x_{r 1}, x_{r 2}\right) \in \mathbf{R}^{2}: x_{r 1} \in \Delta_{r}, a_{r}\left(x_{r 1}\right)-\beta_{r}<x_{r 2}<a_{r}\left(x_{r 1}\right)+\beta_{r}\right\} .
$$

The remaining $W_{M_{0}+1}, \ldots, W_{M}$ can be constructed to have the form (2.1) with each coordinate system $\left(x_{r 1}, x_{r 2}\right)$ a translate of the coordinate functions on $\mathbf{R}^{2}$ and $a_{r}=0$ in such a way that

$$
\bigcup_{r=M_{0}+1}^{M} \bar{W}_{r} \subset \Omega \quad \text { and } \quad \bar{\Omega} \subset \bigcup_{r=1}^{M} W_{r} .
$$

The construction of such coverings of $\bar{\Omega}$ and associated local representation of the boundary of $\Omega$ is standard in the literature (c.f. [4])

We will also need a partition of unity associated with the $W_{r}$-covering. A set of functions $\left\{\varphi_{r}: 1 \leq r \leq M\right\}$ is a partition of unity associated with the 
$W_{r}$-covering if (i) $\varphi_{r} \in C_{0}^{\infty}\left(W_{r}\right)$, (ii) $\varphi_{r} \geq 0$, and (iii) $\sum_{r=1}^{M} \varphi_{r}=1$ on $\bar{\Omega}$. The construction of such partitions of unity is also standard in the literature (c.f. [4]).

In order to study boundary value problems associated with $\Omega$ it will be necessary to approximate $\Omega$ by a domain with smooth boundary. For this purpose, for each $m \in N$ let $\Omega^{(m)}$ be a convex open set with $\Omega^{(m)} \subset \Omega$ and a $C^{\infty}$ boundary such that $\Omega \backslash \Omega^{(m)} \subset N(E, 1 / m)$, and $\partial \Omega \Delta \partial \Omega^{(m)} \subset N(E, 1 / m)$. For large $m$, the local coordinate system $\left(x_{r 1}, x_{r 2}\right)$ can also be used to describe the boundaries of the approximating domains $\Omega^{(m)}$ as follows. For each $r=1, \ldots, M_{0}$ there is a $C^{\infty}$ function $a_{r}^{(m)}: \Delta_{r} \rightarrow \mathbf{R}$ such that each point $x=\left(x_{r 1}, x_{r 2}\right) \in \partial \Omega^{(m)}$ can be represented by $x=\left(x_{r 1}, a_{r}^{(m)}\left(x_{r 1}\right)\right), x_{r 1} \in \Delta_{r}$ for some $r$ and $\left|a_{r}^{(m)}\left(x_{r 1}\right)-a_{r}\left(x_{r 1}\right)\right|<1 / m$ for $x_{r 1} \in \Delta_{r}$. The $a_{r}^{(m)}$ relate to the $\Omega^{(m)}$ in the same way that the $a_{r}$ relate to $\Omega$.

Consider now a denumerably comprehensive enlargement of a structure containing the real numbers $\mathbf{R}$ (see [6] for terminology and notation). For one thing this means that for each $m \in{ }^{*} N$ there is an internal convex domain $\Omega^{(m)} \subset{ }^{*} \Omega$ with an internally $C^{\infty}$ boundary $\partial \Omega^{(m)}$ such that ${ }^{*} \Omega \backslash \Omega^{(m)} \subset N(E, 1 / m)$ and $\partial^{*} \Omega \Delta \partial \Omega^{(m)} \subset N(E, 1 / m)$. Fix $m \in{ }^{*} N \backslash N$ and let $\tilde{\Omega}=\Omega^{(m)}, \tilde{a}_{r}=a_{r}^{(m)}$, $r=1, \ldots, M$. Since $\left\{{ }^{*} \varphi_{r}: 1 \leq r \leq M\right\}$ is a partition of unity for ${ }^{*} \Omega$ and $\tilde{\Omega} \subset{ }^{*} \Omega$, the standard partition of unity $\left\{{ }^{*} \varphi_{r}: 1 \leq r \leq M\right\}$ is a partition of unity for $\tilde{\Omega}$ associated with the ${ }^{*} W_{r}$-covering of $\tilde{\Omega}$. Note also that

$$
\tilde{a}_{r}\left(x_{r 1}\right) \simeq{ }^{*} a_{r}\left(x_{r 1}\right) \quad \text { for all } x_{r 1} \in{ }^{*} \Delta_{r}, r=1, \ldots, M \text {. }
$$

If $S$ is any internal subset of ${ }^{*} \mathbf{R}^{2}$, the set ${ }^{o} S$ is defined by ${ }^{o} S=\left\{x \in \mathbf{R}^{2}\right.$ : there is a $y \in S$ and $y \simeq x\}$ and is closed. In particular, ${ }^{o} \tilde{\Omega}=\bar{\Omega}$. Suppose now that $f$ is an internal function on the internal set $S$ such that $f$ is finite-valued and $s$-continuous on $S$. Then the standard part of $f$ is defined on ${ }^{\circ} S$ as follows. For $x \in{ }^{o} S,{ }^{o} f(x)={ }^{o}[f(y)]$ for $y \in S, y \simeq x$. The function ${ }^{o} f$ is then uniformly continuous on ${ }^{\circ} S$ (c.f. [5], p. 116). Lastly, if $B$ is any internal Banach space then fin $B$ will denote the set of $x \in B$ such that $\|x\|$ is near-standard in ${ }^{*} \mathbf{R}$.

In what follows several inequalities concerning norm estimates of solutions of partial differential equations will be applied to internal, but not standard, domains. This is accomplished by applying the transfer principle with due care being taken to reformulate the theorems so that the transfer principle is applicable in the context used. These inequalities involve constants depending upon various parameters and most of the work that follows involves keeping track of the constants to make sure that they are near-standard.

\section{Existence}

Given a function $f \in C(\bar{\Omega})$ and $\lambda>0$ we would like to prove that there is a function $u \in C^{2}(\Omega)$ which satisfies the equation $\Delta u-\lambda u=f$ on $\Omega$ and the 
boundary condition $\partial u / \partial \nu=0$ on $\partial \Omega \backslash E$. We will accomplish this by considering a more restrictive class of $f$ and the corresponding problem on $\tilde{\Omega}$. Recall that $\mu$ is a fixed number in $(0,1)$.

Lemma 3.1. Given $\lambda>0$ and $f \in C^{0, \mu}(\bar{\Omega})$, there is a unique internal function $u \in C^{2, \mu}(\overline{\tilde{\Omega}})$ with $u \in$ fin $C^{0, \mu}(\overline{\tilde{\Omega}})$ and ${ }^{*} \Delta u \in$ fin $C^{0, \mu}(\overline{\tilde{\Omega}})$ such that

$$
\begin{cases}{ }^{*} \Delta u-\lambda u={ }^{*} f & \text { on } \tilde{\Omega} \\ \frac{\partial u}{\partial \nu}=0 & \text { on } \partial \tilde{\Omega}\end{cases}
$$

in particular, $u$ and ${ }^{*} \Delta u$ are finite-valued and $s$-continuous on $\tilde{\Omega}$.

Proof. Since $f \in C^{0, \mu}(\bar{\Omega}), f$ is bounded and ${ }^{*} f \in C^{0, \mu}(\bar{\Omega})$ (more precisely, the restriction of ${ }^{*} f$ to $\tilde{\Omega}$ is in $\left.C^{0, \mu}(\overline{\tilde{\Omega}})\right)$. It is known that there is a unique internal function $u \in C^{2, \mu}(\overline{\tilde{\Omega}})$ which satisfies (3.1) (see [2], Theorem 6.31 and the comments on p. 124). Since $f$ is bounded,

$$
{ }^{o}\|u\|_{2,2, \tilde{\Omega}} \leq\left(\frac{1}{\lambda^{2}}+\frac{1}{\lambda}+4\right)^{1 / 2}{ }^{o}\left\|^{*} f\right\|_{0,2, \tilde{\Omega}}<\infty
$$

by Theorem 3.1.2.3. of [3]. Letting $p^{\prime}=2 /(2-\mu), 1<p^{\prime}<2$. By Hölder's inequality, there is a constant $C_{1} \in \mathbf{R}$ depending only upon the measure of $\Omega$ and $p^{\prime}$ such that

$$
\|u\|_{2, p^{\prime}, \tilde{\Omega}} \leq C_{1}\|u\|_{2,2, \tilde{\Omega}}
$$

and therefore ${ }^{o}\|u\|_{2, p^{\prime}, \tilde{\Omega}}<\infty$. There is also a constant $C_{2} \in \mathbf{R}$ depending only upon the standard numbers $\left|\varphi_{r}\right|_{0, \bar{\Omega}},\left|D_{i} \varphi_{r}\right|_{0, \bar{\Omega}},\left|D_{i} D_{j} \varphi_{r}\right|_{0, \bar{\Omega}}$ such that

$$
\left\|u^{*} \varphi_{r}\right\|_{2, p^{\prime}, \tilde{\Omega}} \leq C_{2}\|u\|_{2, p^{\prime}, \tilde{\Omega}}
$$

and it follows that ${ }^{o}\left\|u^{*} \varphi_{r}\right\|_{2, p^{\prime}, \tilde{\Omega}}<\infty, r=1,2, \ldots, M$. For $r=1, \ldots, M_{0}$, let

$$
V_{r}=\left\{\left(x_{r 1}, x_{r 2}\right): x_{r 1} \in{ }^{*} \Delta_{r}, \tilde{a}_{r}\left(x_{r 1}\right)<x_{r 2}<\tilde{a}_{r}\left(x_{r 1}\right)+\beta_{r}\right\} .
$$

Since $\varphi_{r}$ vanishes outside a standard compact subset of the open set $W_{r},{ }^{*} \varphi_{r}$ vanishes on $V_{r}^{c} \cap \tilde{\Omega}$. Therefore,

$$
\left\|u^{*} \varphi_{r}\right\|_{2, p^{\prime}, \tilde{\Omega}}=\left\|u^{*} \varphi_{r}\right\|_{2, p^{\prime}, V_{r}}
$$

and ${ }^{o}\left\|u^{*} \varphi_{r}\right\|_{2, p^{\prime}, V_{r}}<\infty$. Consider a fixed $r=1, \ldots, M_{0}$ and let $C=\left\{\left(y_{1}, y_{2}\right) \in\right.$ $\left.\mathbf{R}^{2}:\left|y_{i}\right|<1, i=1,2\right\}$. We now define an internal map $T$ from ${ }^{*} C$ onto $V_{r}$ by writing $x=\left(x_{r 1}, x_{r 2}\right)$ for $x \in V_{r}, y=\left(y_{1}, y_{2}\right)$ for $y \in{ }^{*} C$, and letting

$$
x_{r 1}=\alpha_{r} y_{1}, \quad x_{r 2}=\frac{\beta_{r}}{2} y_{2}+\tilde{a}_{r}\left(\alpha_{r} y_{1}\right)+\frac{\beta_{r}}{2} .
$$


We can also consider $T$ as an internal map from ${ }^{*} \bar{C}$ onto $\bar{V}_{r}$. Since $\tilde{a}_{r}^{\prime}$ is bounded by the maximum of the absolute values of the slopes of adjacent edges of $\partial \Omega$ if $V_{r}$ contains a vertex of $\partial \Omega$ and is equal to the slope of an edge of $\partial \Omega$ if $V_{r}$ does not, there is a constant $C_{3} \in \mathbf{R}$ which depends only upon $\alpha_{r}, \beta_{r}$, and a standard bound on $\tilde{a}_{r}^{\prime}$ such that

$$
\begin{aligned}
& \left|T y^{\prime}-T y^{\prime \prime}\right| \leq C_{3}\left|y^{\prime}-y^{\prime \prime}\right|, \quad y^{\prime}, y^{\prime \prime} \in{ }^{*} \bar{C} \\
& \left|T^{-1} x^{\prime}-T^{-1} x^{\prime \prime}\right| \leq C_{3}\left|x^{\prime}-x^{\prime \prime}\right|, \quad x^{\prime}, x^{\prime \prime} \in \bar{V}_{r}
\end{aligned}
$$

We will use this fact to show that ${ }^{o}\left\|u^{*} \varphi_{r}\right\|_{1, q, V_{r}}<\infty$ where $1 / q=\left(1 / p^{\prime}\right)-(1 / 2)$. Since ${ }^{o}\left\|u^{*} \varphi_{r}\right\|_{2, p^{\prime}, V_{r}}<\infty,{ }^{o}\left\|D^{\alpha}\left(u^{*} \varphi_{r}\right)\right\|_{1, p^{\prime}, V_{r}}<\infty$ for $|\alpha| \leq 1$. Fix $\alpha$ with $|\alpha| \leq 1$ and let $w_{r}(y)=D^{\alpha}\left(u^{*} \varphi_{r}\right)(T y), y \in{ }^{*} \bar{C}$. Since ${ }^{o}\left\|D^{\alpha}\left(u^{*} \varphi_{r}\right)\right\|_{1, p^{\prime}, V_{r}}<\infty$ and the Jacobian of the map $T$ is $\alpha_{r} \beta_{r} / 2,{ }^{o}\left\|w_{r}\right\|_{1, p^{\prime},{ }^{*} \bar{C}}<\infty$. It is shown in the proof of Theorem 3.4 of [4], p. 69, that there is a constant $C_{4} \in \mathbf{R}$ (in fact, $\left.C_{4}=p^{\prime} /\left(2-p^{\prime}\right)\right)$ such that

$$
\left\|w_{r}\right\|_{L_{q}\left({ }^{*} \bar{C}\right)} \leq C_{4}\left\|w_{r}\right\|_{1, p^{\prime},{ }^{*} \bar{C}}
$$

and so ${ }^{o}\left\|w_{r}\right\|_{L_{q}\left({ }^{*} \bar{C}\right)}<\infty$. Using Lemma 3.1 of [4], p. 65 , in conjunction with the transfer principle,

$$
\left\|D^{\alpha}\left(u^{*} \varphi_{r}\right)\right\|_{L_{q}\left(V_{r}\right)} \leq C_{3}\left\|w_{r}\right\|_{L_{q}(* \bar{C})}
$$

and it follows that ${ }^{o}\left\|u^{*} \varphi_{r}\right\|_{1, q, V_{r}}<\infty$. Now let $v_{r}(y)=u^{*} \varphi_{r}(T y), y \varepsilon^{*} \bar{C}$. Again using the fact that the Jacobian of the map $T$ is the standard quantity $\alpha_{r} \beta_{r} / 2$, ${ }^{o}\left\|v_{r}\right\|_{1, q, *^{*} C}<\infty$. By Theorem 3.5 of [4], p. 72 (in particular, the inequality (3.25)), there is a constant $C_{6} \in \mathbf{R}$ such that

$$
|h|_{0, \mu, \bar{C}} \leq C_{6}\|h\|_{1, q, C},
$$

since $\mu=2-\left(2 / p^{\prime}\right)<1$, whenever $h \in W^{1, q}(C)$. By the transfer principle, $|h|_{0, \mu,{ }^{*} \bar{C}} \leq C_{6}\|h\|_{1, q,{ }^{*} \bar{C}}$ whenever $h \in W^{1, q}\left({ }^{*} C\right)$. Therefore, ${ }^{o}\left|v_{r}\right|_{0, \mu,{ }^{*} \bar{C}}<\infty$. Since $|\cdot|_{0, \mu,{ }^{*} \bar{C}}$ includes the term $|\cdot|_{0,{ }^{*} \bar{C}}$, it follows that $v_{r}$, and therefore $u^{*} \varphi_{r}$, is finite-valued. Suppose $x^{\prime}, x^{\prime \prime} \in \bar{V}_{r}, y^{\prime}=T^{-1} x^{\prime}$, and $y^{\prime \prime}=T^{-1} x^{\prime \prime}$. Then

$$
\begin{aligned}
\left|u^{*} \varphi_{r}\left(x^{\prime}\right)-u^{*} \varphi_{r}\left(x^{\prime \prime}\right)\right| & =\left|v_{r}\left(y^{\prime}\right)-v_{r}\left(y^{\prime \prime}\right)\right| \leq\left|v_{r}\right|_{0, \mu,{ }^{*} \bar{C}}\left|y^{\prime}-y^{\prime \prime}\right|^{\mu} \\
& \leq C_{3}^{\mu}\left|v_{r}\right|_{0, \mu,{ }^{*} \bar{C}}\left|x^{\prime}-x^{\prime \prime}\right|^{\mu} .
\end{aligned}
$$

In particular, $x^{\prime} \simeq x^{\prime \prime}$ implies that $u^{*} \varphi_{r}\left(x^{\prime}\right) \simeq u^{*} \varphi_{r}\left(x^{\prime \prime}\right)$ and consequently that $u^{*} \varphi_{r}$ is finite-valued and $s$-continuous on $\tilde{\Omega}$. The latter inequality also shows that $u^{*} \varphi_{r} \in$ fin $C^{0, \mu}\left(V_{r}\right)$. Using the fact that $u^{*} \varphi_{r}$ vanishes on $\tilde{\Omega} \backslash V_{r}$ and the convexity of $\tilde{\Omega}$, it is easily seen that $u^{*} \varphi_{r} \in$ fin $C^{0, \mu}(\tilde{\Omega})$ for $r=1, \ldots, M_{0}$. Clearly the same arguments apply to the $u^{*} \varphi_{r}, r=M_{0}+1, \ldots, M$. Since $u=\sum_{r=1}^{M} u^{*} \varphi_{r}$ on $\overline{\tilde{\Omega}}, u$ is finite-valued and $s$-continuous on $\tilde{\Omega}$ and $u \in$ fin $C^{0, \mu}(\bar{\Omega})$. 
It remains to show that the standard part ${ }^{o} u$ of the internal function $u$ of the preceding lemma satisfies the resolvent equation $\lambda^{o} u-\Delta^{o} u=f$ on $\bar{\Omega}$ and that $\partial^{\circ} u / \partial \nu=0$ on $\delta \Omega \backslash E$.

Consider an open interval $I \subset \mathbf{R}, x \in I$, and $g \in$ fin $C^{1, \mu}\left({ }^{*} I\right)$. Then $g$ and $g^{\prime}$ are both finite-valued and $s$-continuous on ${ }^{*} I$ so that ${ }^{o} g$ and ${ }^{o}\left(g^{\prime}\right)$ are both defined on $\bar{I}$. We claim that ${ }^{o}\left(g^{\prime}(x)\right)=\left({ }^{o} g\right)^{\prime}(x)$. To see this, let $h$ be a standard positive number with $[x-2 h, x+2 h] \subset I$. For any standard positive number $\varepsilon$

$$
\begin{aligned}
\left|\frac{{ }^{o} g(x+h)-{ }^{o} g(x)}{h}-{ }^{o}\left(g^{\prime}(x)\right)\right| & \leq\left|\frac{g(x+h)-g(x)}{h}-g^{\prime}(x)\right|+\varepsilon \\
& =\left|g^{\prime}(x+\theta h)-g^{\prime}(x)\right|+\varepsilon \\
& \leq\left(|g|_{1, \mu}, *_{I}\right)|\theta h|^{\mu}+\varepsilon \\
& \leq\left(|g|_{1, \mu}, *_{I}\right)|h|^{\mu}+\varepsilon
\end{aligned}
$$

where $\theta \in{ }^{*}[0,1]$. Since ${ }^{o}|g|_{1, \mu}, *_{I}<\infty$ and $\varepsilon$ is arbitrary, $\left({ }^{o} g\right)^{\prime}(x)={ }^{o}\left(g^{\prime}(x)\right)$.

Suppose now that $I \subset \mathbf{R}^{2}$ is an open rectangle, $(x, y) \in I$, and $g \in$ fin $C^{2, \mu}\left({ }^{*} I\right)$. Then $g, \partial g / \partial x$, and $\partial^{2} g / \partial x^{2}$ are all finite-valued and $s$-continuous on ${ }^{*} I$, and ${ }^{o} g,{ }^{o}(\partial g / \partial x),{ }^{o}\left(\partial^{2} g / \partial x^{2}\right)$ are defined on $\bar{I}$. Repeating the above argument for $\partial\left({ }^{\circ} g\right) / \partial x$ it is easily seen that

$$
{ }^{\circ}\left(\frac{\partial^{2} g}{\partial x^{2}}\right)(x, y)=\frac{\partial^{2}\left({ }^{o} g\right)}{\partial x^{2}}(x, y)
$$

with similar results holding for the other second partials.

Before applying the last result, we will need some additional notation. If $S$ is a bounded open subset of $\mathbf{R}^{2}$ and $x, y \in S$, let $d_{x}=\operatorname{dist}(x, \partial S) \leq \operatorname{diam}(S)$ and let $d_{x, y}=\min \left(d_{x}, d_{y}\right)$. For $\sigma \in \mathbf{R}$, a nonnegative integer $k$, and $u \in C^{k}(\Omega)$, let

$$
\begin{aligned}
& {[u]_{k, S}^{(\sigma)}=\sup _{x \in S,|\beta|=k} d_{x}^{k+\sigma}\left|D^{\beta} u(x)\right|} \\
& |u|_{k, S}^{(\sigma)}=\sum_{j=0}^{k}[u]_{j, S}^{(\sigma)}
\end{aligned}
$$

if $u \in C^{k, \alpha}(\Omega), 0<\alpha \leq 1$, let

$$
\begin{aligned}
& {[u]_{k, \alpha, S}^{(\sigma)}=\sup _{x, y \in S,|\beta|=k} d_{x, y}^{k+\alpha+\sigma} \frac{\left|D^{\beta} u(x)-D^{\beta} u(y)\right|}{|x-y|^{\alpha}}} \\
& |u|_{k, \alpha, S}^{(\sigma)}=|u|_{k, S}^{(\sigma)}+[u]_{k, \alpha, S}^{(\sigma)} .
\end{aligned}
$$

Note that

$$
|u|_{0, S}^{(2)}=[u]_{0, S}^{(2)}=\sup _{x \in S} d_{x}^{2}|u(x)| \leq(\operatorname{diam} S)^{2}|u|_{0, S}
$$


and

$$
\begin{aligned}
|\mu|_{0, \alpha, S}^{(2)} & =[u]_{0, S}^{(2)}+[u]_{0, \alpha, S}^{(2)} \\
& \leq(\operatorname{diam} S)^{2}|u|_{0, S}+(\operatorname{diam} S)^{\alpha+2}[u]_{0, \alpha, S} \\
& \leq\left\{(\operatorname{diam} S)^{2}+(\operatorname{diam} S)^{\alpha+2}\right\}|\mu|_{0, \alpha, S}
\end{aligned}
$$

If, in addition, $T$ is a $C^{k, \alpha}$ portion of the boundary of $S$, we put $\bar{d}_{x}=\operatorname{dist}(x, \partial S \backslash$ $T), \bar{d}_{x, y}=\min \left(\bar{d}_{x}, \bar{d}_{y}\right)$ and for $u \in C^{k, \alpha}(S \cup T)$ we define

$$
\begin{aligned}
{[u]_{\hat{k}, S \cup T} } & =\sup _{x \in S,|\beta|=k} \bar{d}_{x}^{k}\left|D^{\beta} u(x)\right| \\
{[u]_{k, \alpha, S \cup T} } & =\sup _{x, y \in S \cup T,|\beta|=k} \bar{d}_{x, y}^{k+\alpha} \frac{\left|D^{\beta} u(x)-D^{\beta} u(y)\right|}{|x-y|^{\alpha}} \\
|u|_{k, S \cup T} & =\sum_{j=0}^{k}[u]_{j, S \cup T} \\
|u|_{k, \alpha, S \cup T} & =|u|_{k, S \cup T}+[u]_{k, \alpha, S \cup T} .
\end{aligned}
$$

In order to avoid overuse of "*", we have chosen to use " " " rather than the "*" as in [2]. We also define $\mathscr{D}(\Delta)$ to be the set of $u \in C(\bar{\Omega}) \cap C^{2}(\Omega)$ for which there is a $v \in C^{2, \mu}(\overline{\tilde{\Omega}}) \cap$ fin $C^{0, \mu}(\tilde{\tilde{\Omega}})$ with ${ }^{*} \Delta v \in$ fin $C^{0, \mu}(\tilde{\tilde{\Omega}})$ and $\partial v / \partial \nu=0$ on $\partial \tilde{\Omega}$ such that $u={ }^{o} v$ and $\Delta u={ }^{o}\left({ }^{*} \Delta v\right)$. It is easily seen that $u \in C^{0, \mu}(\bar{\Omega})$ and $\Delta u \in C^{0, \mu}(\bar{\Omega})$. The set of $v \in C^{2, \mu}(\overline{\tilde{\Omega}})$ defining $\mathscr{D}(\Delta)$ is, of course, an external set.

Lemma 3.2. Given $\lambda>0$ and $f \in C^{0, \mu}(\bar{\Omega})$, there is a $u \in \mathscr{D}(\Delta)$ such that $\Delta u-\lambda u=f$ on $\bar{\Omega}$ and $\partial u / \partial \nu=0$ on $\partial \Omega \backslash E$.

Proof. Let $v \in C^{2, \mu}(\overline{\tilde{\Omega}})$ be the internal function of Lemma 3.1 with $v \in$ fin $C^{0, \mu}(\overline{\tilde{\Omega}})$ and ${ }^{*} \Delta v \in$ fin $C^{0, \mu}(\overline{\tilde{\Omega}})$ and let $u={ }^{o} v$ on $\bar{\Omega}$. Let $(x, y)$ be an interior point of $\Omega$ and let $\Omega^{\prime}$ be a disk centered at $(x, y)$ such that $d\left(\Omega^{\prime}, \partial \Omega\right)=\delta>0$. Let $\tilde{\delta}=d\left({ }^{*} \Omega^{\prime}, \partial \tilde{\Omega}\right) \leq \delta$ with $\tilde{\delta} \simeq \delta$. By the transfer principle and Corollary 6.3 of [2], there is a standard constant $C(\mu)$, depending only upon $\mu$, such that

$$
\tilde{\delta}|D v|_{0, * \Omega^{\prime}}+\tilde{\delta}^{2}\left|D^{2} v\right|_{0, * \Omega^{\prime}}+\tilde{\delta}^{2+\mu}\left[D^{2} v\right]_{\mu, * \Omega^{\prime}} \leq C(\mu)\left(|v|_{0, \tilde{\Omega}}+\left.\left.\right|^{*} f\right|_{0, \mu, \tilde{\Omega}} ^{(2)}\right)
$$

By (3.4),

$$
\begin{aligned}
\left.\left.\right|^{*} f\right|_{0, \mu, \tilde{\Omega}} ^{(2)} & \leq\left.\left.\left\{(\operatorname{diam} \tilde{\Omega})^{2}+(\operatorname{diam} \tilde{\Omega})^{\mu+2}\right\}\right|^{*} f\right|_{0, \mu, \tilde{\Omega}} \\
& \leq\left\{(\operatorname{diam} \Omega)^{2}+(\operatorname{diam} \Omega)^{\mu+2}\right\}|f|_{0, \mu, \Omega}
\end{aligned}
$$

and $\left.\left.{ }^{o}\right|^{*} f\right|_{0, \mu, \bar{\Omega}} ^{(2)}<\infty$. Since $\tilde{\delta}>\delta / 2,{ }^{o}|D v|_{0, *^{*} \Omega^{\prime}}<\infty,{ }^{o}\left|D^{2} v\right|_{0,{ }^{*} \Omega^{\prime}}<\infty$, and ${ }^{o}\left[D^{2} v\right]_{\mu, * \Omega^{\prime}}<\infty$. Since ${ }^{o}|v|_{0, * \Omega^{\prime}} \leq{ }^{o}|v|_{0, \bar{\Omega}}<\infty$, it follows that $v \in$ fin $C^{2, \mu}\left({ }^{*} \Omega^{\prime}\right)$ 
since $|v|_{2, \mu, * \Omega^{\prime}}$ is the sum of these four near-standard quantities. Therefore, $\Delta u(x, y)=\Delta\left({ }^{o} v\right)(x, y)={ }^{o}\left({ }^{*} \Delta v\right)(x, y)$. Since ${ }^{*} \Delta v-\lambda v={ }^{*} f, \Delta u(x, y)-$ $\lambda u(x, y)=f(x, y)$ and $\Delta u-\lambda u=f$ on $\Omega$. The latter fact implies that $\Delta u$ has a continuous extension to $\bar{\Omega}$ since $f$ and $u$ are continuous on $\bar{\Omega}$. Now consider any $x \in \partial \Omega \backslash E$. Let $\varrho=d(x, E), \Sigma=B_{x, \varrho / 2} \cap \Omega$, and $T=\bar{B}_{x, \varrho / 4} \cap \partial \Omega$. Since $v \in C^{2, \mu}(\overline{\tilde{\Omega}}), v \in C^{2, \mu}\left({ }^{*} \Sigma \cup^{*} T\right)$ and ${ }^{*} \Delta v-\lambda v={ }^{*} f$ on ${ }^{*} \Sigma$. By the transfer principle and Lemma 6.29 of [2],

$$
|v|_{2, \mu, * \Sigma U^{*} T} \leq C(\mu, \varrho)\left(|v|_{0, * \Sigma}+\left.\left.\right|^{*} f\right|_{0, \mu, * \Sigma}\right)
$$

where $C(\mu, \varrho)$ is a standard constant depending only upon $\mu$ and $\varrho$. Since $|v|_{0, * \Sigma} \leq|v|_{0, \bar{\Omega}}$ and the latter is near-standard, ${ }^{o}|v|_{0, * \Sigma}<\infty ;$ likewise, $\left.\left.{ }^{o}\right|^{*} f\right|_{0, \mu, * \Sigma}$ $\leq\left.\left.^{o}\right|^{*} f\right|_{0, \mu, \Omega}=|f|_{0, \mu, \Omega}<\infty$. Therefore, ${ }^{o}|v|_{2, \mu},{ }^{*} \Sigma \cup^{*} T<\infty$. For any standard $\varepsilon$, $t>0$,

$$
\begin{aligned}
\left|\frac{v(x-t \nu)-{ }^{o} v(x)}{-t}-{ }^{o}\left(\frac{\partial v}{\partial \nu}(x)\right)\right| & \leq\left|\frac{v(x-t \nu)-v(x)}{-t}-\frac{\partial v}{\partial \nu}(x)\right|+\varepsilon \\
& =|\nabla v(z) \cdot \nu-\nabla v(x) \cdot \nu|+\varepsilon
\end{aligned}
$$

where $z$ is a point on the line segment from $x$ to $x-t \nu$. For $t<\varrho / 4$, after applying the mean value theorem again and noting that $\bar{d}_{z} \geq \varrho / 4$ for any point $z$ on the line segment joining $x-(\varrho / 4) \nu$ to $x$,

$$
\left|\frac{o v(x-t \nu)-{ }^{o} v(x)}{-t}-{ }^{o}\left(\frac{\partial v}{\partial \nu}(x)\right)\right| \leq 64 \varrho^{-2} \mid v \hat{2}_{2, \mu, * \Sigma U^{*} T} t+\varepsilon .
$$

Therefore,

$$
\frac{\partial u}{\partial \nu}(x)=\frac{\partial^{o} v}{\partial \nu}(x)={ }^{\circ}\left(\frac{\partial v}{\partial \nu}(x)\right)=0 .
$$

This completes the proof that $u \in \mathscr{D}(\Delta)$.

We can now prove the main result.

Theorem 3.3. The Laplacian $\Delta$ with domain $\mathscr{D}(\Delta)$ is closable and its minimal closed extension $A$ generates a Feller semigroup $\left\{T_{t}: t \geq 0\right\}$ on $C(\bar{\Omega})$.

Proof. We need only verify (i), (ii), (iii) of $(1,1)$. Since $C^{0, \mu}(\bar{\Omega})$ contains the polynomial functions restricted to $\bar{\Omega}$, which are dense in $C(\bar{\Omega})$, the range of $\Delta-\lambda$ is dense in $C(\bar{\Omega})$ for all $\lambda>0$ by Lemma 3.2. We now show that $\Delta$ with domain $\mathscr{D}(\Delta)$ is dissipative. Consider the operator ${ }^{*} \Delta$ with domain

$$
\tilde{\mathscr{D}}\left({ }^{*} \Delta\right)=\left\{w: w \in C^{0}(\overline{\tilde{\Omega}}),{ }^{*} \Delta w \in C^{0}(\overline{\tilde{\Omega}}), \partial w / \partial \nu=0 \text { on } \partial \tilde{\Omega}\right\} .
$$


It is known that ${ }^{*} \Delta$ with this domain has a minimal closed extension which is the infinitesimal generator of a Feller semigroup $\left\{\tilde{T}_{t}: t \geq 0\right\}$ on $C^{0}(\tilde{\Omega})$ (c.f. [8]). As a consequence, ${ }^{*} \Delta$ with domain $\tilde{\mathscr{D}}\left({ }^{*} \Delta\right)$ is dissipative. Suppose $u \in \mathscr{D}(\Delta)$ and let $v \in C^{2, \mu}(\overline{\tilde{\Omega}})$ be the internal function with $v \in$ fin $C^{0, \mu}(\overline{\tilde{\Omega}})$ and ${ }^{*} \Delta v \in$ fin $C^{0, \mu}(\overline{\tilde{\Omega}})$ such that $u={ }^{o} v, \Delta u={ }^{o}\left({ }^{*} \Delta v\right)$, and $\partial v / \partial \nu=0$ on $\partial \tilde{\Omega}$. Then $v \in \tilde{\mathscr{D}}\left({ }^{*} \Delta\right)$ and therefore

$$
\left.\right|^{*} \Delta v-\left.\lambda v\right|_{0, \tilde{\Omega}} \geq \lambda|v|_{0, \bar{\Omega}}
$$

from which it follows that $|\Delta u-\lambda u| \geq \lambda|u|$. Thus $\Delta$ with domain $\mathscr{D}(\Delta)$ is dissipative. To complete the proof we need only show that $\mathscr{D}(\Delta)$ is dense in $C(\bar{\Omega})$. Let $\mathscr{D}_{0}$ be the set of functions $u \in C^{\infty}(\bar{\Omega})$ which are constant on a neighborhood of each vertex of $\Omega$ and $\partial u / \partial \nu=0$ on $\partial \Omega \backslash E$. $\mathscr{D}_{0}$ is an algebra of functions which contains the constant functions. By the Stone-Weierstrass Theorem we need only show that $\mathscr{D}_{0}$ separates the points of $\bar{\Omega}$ to show that $\mathscr{D}_{0}$ is dense in $C(\bar{\Omega})$. Consider two distinct points of $\bar{\Omega}$. If one of them is an interior point of $\Omega$, then there is a $C^{\infty}(\bar{\Omega})$ function in $\mathscr{D}_{0}$ taking on the value 1 at the interior point and vanishing outside a closed disc in the interior of $\Omega$ which does not contain the other point. We need only consider the case that both points belong to $\partial \Omega$. For notational convenience we will assume that one of them is $(0,0)$. For $0<\varrho<1$, let $\varphi_{\varrho}: \mathbf{R} \rightarrow \mathbf{R}$ be any $C^{\infty}(\mathbf{R})$ function which is equal to 1 on $\left(-\varrho^{2} / 4, \varrho^{2} / 4\right)$, equal to zero outside $\left(-\varrho^{2}, \varrho^{2}\right)$, and $0 \leq \varphi_{\varrho} \leq 1$. If we put $u_{\varrho}(x, y)=\varphi_{\varrho}\left(x^{2}+y^{2}\right)$ for $(x, y) \in \bar{\Omega}$, then $u_{\varrho} \in C^{\infty}(\bar{\Omega})$. For sufficiently small $\varrho$, the function $u_{\varrho}$ belongs to $\mathscr{D}_{0}$ and separates the two points under consideration, taking into account the two cases in which $(0,0)$ is a vertex and $(0,0)$ is an interior point of an edge. Consider any $u \in \mathscr{D}_{0}$. The restriction of ${ }^{*} u$ to $\overline{\tilde{\Omega}}$ satisfies the conditions stated just prior to Lemma 3.2 defining an element of $\mathscr{D}(\Delta)$. Since ${ }^{o}\left({ }^{*} u\right)=u, u \in \mathscr{D}(\Delta)$. Thus $\mathscr{D}_{0} \subset \mathscr{D}(\Delta)$ and since $\mathscr{D}_{0}$ is dense in $C(\bar{\Omega}), \mathscr{D}(\Delta)$ is dense in $C(\bar{\Omega})$.

\section{References}

[1] Ethier, S.N., and T.G. KurTz: Markov processes. Characterization and convergence. John Wiley and Sons, New York, 1986.

[2] Gilbarg, D., and N.S. Trudinger: Elliptic partial differential equations of second order. - Springer-Verlag, New York, 1977.

[3] Grisvard, P.: Elliptic problems in nonsmooth domains. - Pitman Publishing, Inc., Marshfield, Massachusetts, 1985.

[4] NeČas, J.: Les méthodes directes en théorie des équations elliptiques. - Masson et $\mathrm{C}^{\text {ie }}$, Éditeurs, Paris, 1967.

[5] Robinson, A.: Non-standard analysis, Revised edition. - North-Holland Publishing Company, Amsterdam, 1974.

[6] Stroyan, K., and W.A.J. Luxemburg: Introduction to the theory of infinitesimals. Academic Press, New York, 1976.

[7] TANAKA, H.: Stochastic differential equations with reflecting boundary condition in convex regions. - Hiroshima Math. J. 9, 1979, 163-177. 
[8] TaIRa, K.: Diffusion processes and partial differential equations. - Academic Press, London, 1988.

[9] VaradhaN, S.R.S., and R.J. Williams: Brownian motion in a wedge with oblique reflection. - Comm. Pure Appl. Math. 38, 1985, 405-443.

[10] Williams, R.J.: Reflected Brownian motion with skew symmetric data in a polyhedral domain. - Probab. Theory Related Fields 25, 1987, 459-485.

Received 2 December 1991 